\title{
Impacto de las Tecnologías de la Información y la Comunicación (Conectivismo) en la Promoción de Vivienda. Un estudio comparativo en la ciudad de Quito y Portoviejo - Ecuador
}

\section{Impact of Information and Communication Technologies (Conectivismo) on the Promotion of Housing. A comparative study in the city of Quito and Portoviejo - Ecuador}

\author{
Mónica Elva Vaca-Cárdenas ${ }^{1 *}$, Ermenson Ricardo Ordoñez Ávila ${ }^{1}$, Astrid Astromelia Vargas Estrada ${ }^{2}$, Leticia \\ Azucena Vaca-Cárdenas ${ }^{1}$ \\ ${ }^{1}$ Universidad Técnica de Manabí \\ 2 Escuela Superior Politécnica de Chimborazo \\ *monivcec@yahoo.com
}

DOI: https://doi.org/10.26871/killkana_tecnica.v3i3.587

\begin{abstract}
Resumen
La sociedad actual se ha visto influenciada por la proliferación de las tecnologías de la información y comunicación (TIC). La evolución de estas tecnologías ha facilitado el acceso a todo tipo de información provocando un aumento considerable de la interactividad entre personas de distintos lugares del mundo. Adicionalmente, el uso de la tecnología permite ser más eficientes en el uso de recursos, vivienda, mejorar los servicios, y solucionar los transcendentales problemas de los ciudadanos. El ámbito en que esta investigación se enfoca es el impacto de la tecnología en la promoción de vivienda. Consecuentemente, el objetivo de esta investigación es analizar el impacto de las Tecnologías de la Información y la Comunicación (Conectivismo) en la Promoción de Vivienda en la ciudad de Quito y Portoviejo - Ecuador. Esta investigación fue un estudio comparativo entre Quito y Portoviejo. Se aplicó una encuesta dirigida a 319 propietarios de vivienda en venta o en arriendo en la ciudad de Quito y Portoviejo - Ecuador. De las cuales, 232 encuestas fueron realizadas en la ciudad de Quito y 87 encuestas fueron realizadas en la ciudad de Portoviejo. El análisis de resultados se lo realizó aplicando minería de texto y con la utilización de la herramienta estadística del estudio R. Los resultados de esta investigación han demostrado que el uso de medios tecnológicos contribuye predominantemente para ofertar vivienda en las ciudades de Quito y Portoviejo, por parte de propietarios de la misma. Así, se concluye que, las Tecnologías de la Información y la Comunicación (Conectivismo) están impactando la promoción de vivienda en las ciudades de Quito y Portoviejo -Ecuador.
\end{abstract}

Palabras clave: Tecnologías de la Información y Comunicación, Conectivismo, Promoción de Vivienda.

\begin{abstract}
Today's society has been influenced by the proliferation of information and communication technologies (ICT). The evolution of these technologies has facilitated the access to all kinds of information, causing a considerable increase in the interactivity among people from different parts of the world. Additionally, the use of technology allows us to be more efficient in the use of resources, housing, improve services, and solve the transcendental problems of citizens. The area in which this research focuses is the impact of technology on housing promotion. Consequently, the objective of this research is to analyze the impact of Information and Communication Technologies (Connectivism) on the Promotion of Housing in the city of Quito and Portoviejo - Ecuador. This investigation was a comparative study between Quito and Portoviejo. A survey was applied to 319 homeowners for sale or rent in the city of Quito and Portoviejo - Ecuador. From which, 232 surveys were conducted in the city of Quito and 87 surveys were conducted in the city of Portoviejo. The analysis of results was carried out applying text mining and the use of the statistical tool of study $R$. The results of this research have shown that the use of technological means predominantly contributes to offer housing in the cities of Quito and Portoviejo. Thus, it is concluded that the Information and Communication Technologies (Connectivism) are impacting the promotion of housing in the cities of Quito and Portoviejo - Ecuador.
\end{abstract}

Keywords: Information and Communication Technology, Connectivism, Housing. 


\section{INTRODUCCIÓN}

La humanidad actual vive una revolución tecnológica vertiginosa. Esta revolución se da en buena parte debido a los avances significativos de las Tecnologías de la Información y la Comunicación (TIC). A cause del acelerado crecimiento poblacional en las ciudades a nivel mundial y en nuestro país, y a la vez, a causa del desarrollo tecnológico; los procesos de urbanización y organización también necesitan evolucionar.

Existen posibilidades extraordinarias de utilizar las Tecnologías de la Información y la Comunicación (TIC) para enfrentar los desafíos que tienen las ciudades [1]. Ciertamente, muchos de los principales problemas que las ciudades enfrentan hoy en día pueden ser resueltos con el uso de las Tecnologías de la Información y la Comunicación a través del Conectivismo, que es el vínculo que conecta las nuevas tecnologías con los ciudadanos en ciudades cognitivas. El uso de la tecnología permite ser más eficientes en el uso de recursos, energía, vivienda, mejorar los servicios, y solucionar los transcendentales problemas de los ciudadanos $[2,3]$.

Existe falta de investigación sobre la oferta de vivienda en Ecuador y específicamente sobre la forma en que los propietarios de vivienda ofertan su vivienda para la venta o arrendamiento en el país. Consecuentemente, el objetivo de esta investigación es analizar el impacto de las Tecnologías de la Información y la Comunicación (Conectivismo) en la Promoción de Vivienda en la ciudad de Quito y Portoviejo - Ecuador.

Dentro de la literatura, empezamos describiendo lo que es una ciudad. Una ciudad es un lugar topográficamente descriptible y geográficamente definible con características, asentamientos e infraestructuras que separan a la ciudad o área urbana del área rural [4]. Ciudad es un lugar donde un gran número de diferentes personas viven, trabajan, crean formas de vida específicas y, desarrollan una identidad común [5].

La ciudad inteligente en cambio, adquiere la idea básica de que la función relevante de la ciudad se enriquece con las Tecnologías de la Información y la Comunicación y puede contribuir a desarrollar de manera eficiente y sostenible el diseño socio ecológico del espacio urbano [6]. En general, el uso de Internet y servicios basados en la web, están destinados a ayudar a que las ciudades puedan desarrollarse, y convertirse en lugares, más hermosos y más viables.

Concisamente, las concepciones y proyectos de ciudades inteligentes tienden a centrarse en la mejora de la eficiencia y la sostenibilidad, específicamente con respecto a la vivienda, a la movilidad, a la seguridad, a la protección del medio ambiente y clima, a la gestión de residuos, al uso eficiente de la energía, al uso eficiente de los recursos naturales como el agua, a los servicios de administración municipal, entre otros [7, 8].

Por ende, es imperativo que los ciudadanos tengan el acceso legítimo a participar en la organización y proyectos de sus ciudades y comunidades desde su percepción humana [9]. En este sentido, nace el enfoque de Ciudad Cognitiva, donde para abordar los requerimientos de la sociedad, utiliza la inteligencia urbana o la inteligencia colectiva de los ciudadanos [10].

Es decir, el concepto de ciudad cognitiva combina la inteligencia de los seres humanos y la Inteligencia Artificial (IA) o dispositivos electrónicos [11]. El super -pegamento que conecta ambos elementos (la participación de las nuevas tecnologías y el factor humano) puede describirse como çonectivismo, teoría de aprendizaje y cognición para la era digital, que constituye el vínculo que conecta la tecnología y la inteligencia humana para crear ciudades cognitivas.

La evolución de las Tecnologías de la Información y la Comunicación ha facilitado el acceso a todo tipo de información provocando un aumento considerable de la interactividad entre personas de distintos lugares del mundo. El ámbito en que esta investigación se enfoca es el impacto de la tecnología en la promoción de vivienda en Quito y Portoviejo, como evidencia que los ciudadanos aplican el Conectivismo en pro de crear ciudades cognitivas.

A continuación, se presenta la Metodología, los Resultados, y la Conclusión.

\section{Metodología}

Esta investigación es un anàlisis comparativo del impacto de las Tecnologías de la Información y la Comunicación (Conectivismo) en la Promoción de Vivienda en la ciudad de Quito y Portoviejo - Ecuado. Este artículo analiza los datos extraídos de 2 encuestas realizadas separadamente en las dos ciudades.

La pregunta principal de la encuesta fue una pregunta abierta, a través de la cual se recogió la opinión de los ciudadanos para la caracterización de los datos. La pregunta seleccionada para este estudio fue:¿Por qué medio o plataforma decidió poner en oferta su vivienda o departamento?, Dicha pregunta fue dirigida a 232 propietarios de vivienda en venta o arriendo en la ciudad de Quito y a 87 propietarios de vivienda en venta o arriendo en la ciudad de Portoviejo, dando un total de 319 participantes.

\section{RESUlTADOS}

Los siguientes fueron los resultados de la aplicación de la pregunta abierta: ¿Por qué medio o plataforma decidió poner en oferta su vivienda o departamento?, obtenidos de 319 propietarios de vivienda en venta o en arriendo en la ciudad de Quito y Portoviejo - Ecuador

De 232 encuestados en Quito, la tabla I muestra el rango de edades y el género de los ciudadanos encuestados. Se reporta que de los propietarios de vivienda, la mayoría son de sexo masculino con un total de 122 hombres versus 110 mujeres. Además se observa que la mayoría de reportes provienen de participantes comprendidos entre las edades de 30 y 40 años en hombres y entre 25 y 30 años de edad en mujeres.

La tabla II muestra el rango de edades y el género de 87 ciudadanos encuestados en Portoviejo. Se reporta que 
de los propietarios de vivienda encuestados, la mayoría son de sexo masculino con un total de 58 hombres versus 29 mujeres. Al igual que en Quito, se observa también que la mayoría de reportes provienen de participantes comprendidos entre las edades de 30 y 40 años, en hombres y en mujeres en edades comprendidas entre 25 y 30.

TABLA I: Participación de encuestados por edades y sexo. Quito

\begin{tabular}{|c|c|c|c|c|}
\hline Id & Edad & & Hombres & Mujeres \\
\hline 1 & $25-30$ & & 25 & 34 \\
\hline 2 & $30-40$ & & 48 & 30 \\
\hline 3 & $40-50$ & & 31 & 28 \\
\hline 4 & $50-60$ & & 18 & 18 \\
\hline & Total & & $\mathbf{1 2 2}$ & $\mathbf{1 1 0}$ \\
\hline
\end{tabular}

Fuente: Elaboración propia

TABLA II: Participación de encuestados por edades y sexo. Portoviejo

\begin{tabular}{|c|c|c|c|}
\hline Id & Edad & Hombres & Mujeres \\
\hline 1 & $25-30$ & 16 & 12 \\
\hline 2 & $30-40$ & 21 & 9 \\
\hline 3 & $40-50$ & 10 & 6 \\
\hline 4 & $50-60$ & 11 & 2 \\
\hline & Total & $\mathbf{5 8}$ & $\mathbf{2 9}$ \\
\hline
\end{tabular}

Fuente: Elaboración propia

En cuanto a la frecuencia de medios, el gráfico 1 presenta el reporte univariado de los medios más usados por los oferentes de viviendas o departamentos en venta o en arriendo con el mayor número de repeticiones en la ciudad de Quito. El gráfico 1 reporta el uso de internet con el el porcentaje más alto de $24.6 \%$. A continuación se visualiza a Redes Sociales con $11.2 \%$, seguido de OLX con el $1.3 \%$, Bienes raíces con el $9.1 \%$, Carteles con el $8.2 \%$, Facebook con el $6.9 \%$, Periódico con el 6\%, Volantes con el $5.6 \%$, Anuncios con el $5.2 \%$, Personalmente con el $4.3 \%$, Plataformas con el $3.4 \%$, y finalmente, con el porcentaje más bajo se visualiza Twitter y Airbnb con el $2.6 \%$.

Por otro lado, en la ciudad de Portoviejo, también la opción internet presenta el más alto porcentaje. El gráfico 2 reporta el uso de internet con $25.3 \%$, seguido por Redes sociales con $13.8 \%$, Facebook con $11.5 \%$, Carteles con $9.2 \%$, OLX con $6.9 \%$ al igual que Anuncios, Personalmente con $5.8 \%$, Broker con $5.7 \%$, Plataformas y Bienes Raíces con $4.6 \%$, Periódicos con $3.4 \%$, y Volantes con el $2.3 \%$. Siendo Volantes la opción menos utilizada por los encuestados.

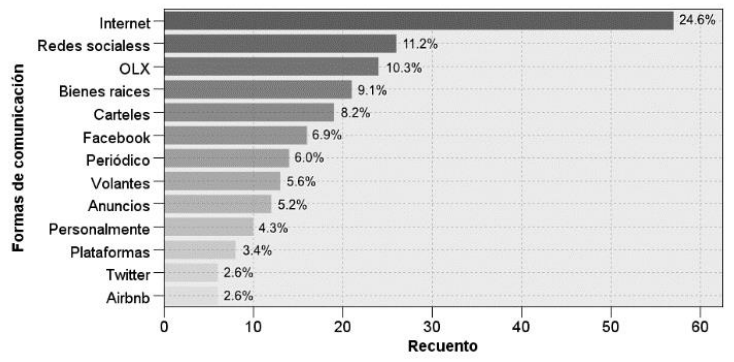

Fig. 1: Frecuencia de medios reportados en Quito Fuente: Elaboración propia

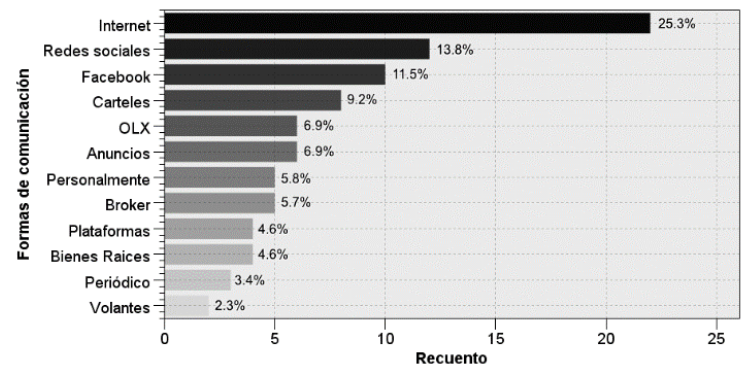

Fig. 2: Frecuencia de medios reportados en Portoviejo Fuente: Elaboración propia

Se procedió a agrupar las diferentes variables de medios en dos categorías con base en la referencia de la frecuencia de palabras. En el gráfico 3 se evidencia que en la ciudad de Quito el uso de Internet alcanza el $61.6 \%$ superando al grupo de Otros que reporta el $38.4 \%$.

La ciudad de Portoviejo también evidencia superioridad en el uso de Internet. El gráfico 4 muestra que el uso del internet destaca con el $62.1 \%$, versus el $37.9 \%$. de uso de medios tradicionales.

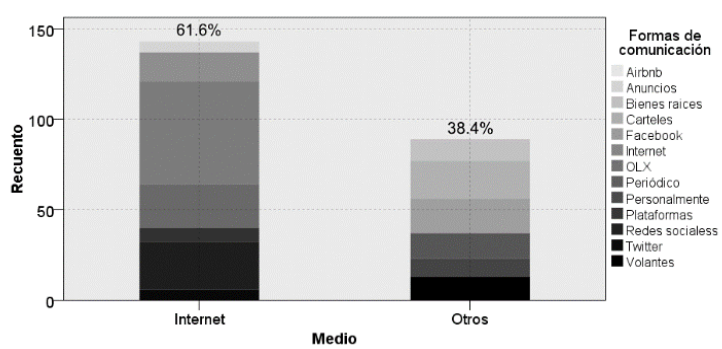

Fig. 3: Internet y Otros - Quito

Fuente: Elaboración propia 


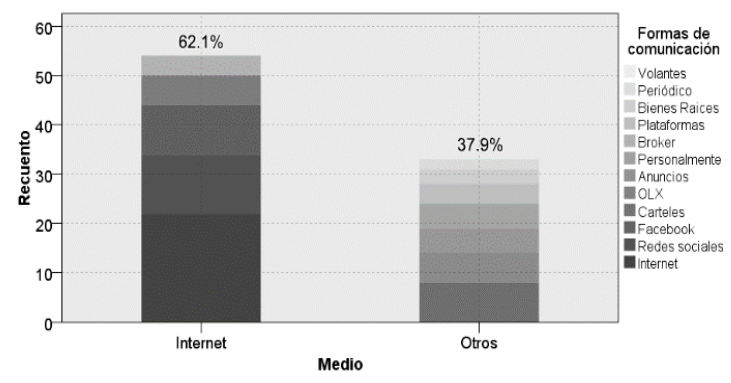

Fig. 4: Internet y Otros - Portoviejo

Fuente: Elaboración propia

Finalmente se realizó un análisis comparativo de las dos ciudades, obteniendo los siguientes reportes:

TABLA III: Participación de encuestados por edad y sexo. Resumen Quito y Portoviejo

\begin{tabular}{|c|c|c|c|}
\hline Edad & H & M & H+M \\
\hline $25-30$ & 41 & 46 & 87 \\
\hline $30-40$ & 69 & 39 & 108 \\
\hline $40-50$ & 41 & 34 & 75 \\
\hline $50-60$ & 29 & 20 & 49 \\
\hline Total & $\mathbf{1 8 0}$ & $\mathbf{1 5 9}$ & $\mathbf{3 1 9}$ \\
\hline
\end{tabular}

Fuente: Elaboración propia

Al sintetizar los datos de el rango de edades y el género de los ciudadanos encuestados, la tabla III visualiza que la mayoría de encuestados propietarios de vivienda son de sexo masculino con un total de 180 hombres versus 159 mujeres. Además se observa que la mayoría de reportes provienen de participantes comprendidos entre las edades de 30 y 40 años tanto en hombres como en mujeres.

En cuanto a los medio má usados para ofertar vivienda en venta o en arriendo, el gráfico 5 muestar los datos integrados de las dos ciudades. Se reporta al Internet liderando con $24.8 \%$, seguido de Redes sociales con $11.9 \%$, OLX con $9.4 \%$, Carteles con $8.5 \%$, Facebook con $8.1 \%$, Bienes raíces con $7.8 \%$, Anuncios con $5.6 \%$, Periódico con $5.3 \%$, Volantes y Personalmente con $4.7 \%$, Plataformas con $3.8 \%$, Twitter y Airbnb con $1.9 \%$ y finalmente con el menor porcentaje, Broker con $1.6 \%$.

El uso de internet supera en gran proporción a las demas opciones mencionaldas por los encuestados. Sin embargo es importante mencionar que Carteles, un medio tradicional tiene un alto porcentaje, ocupando el 4to lugar entre las opciones mencionados

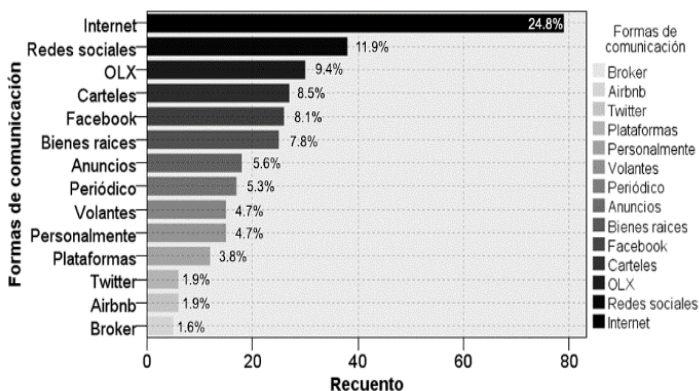

Fig. 5: Resumen de frecuencia de medios reportados en Quito y Portoviejo

Fuente: Elaboración propia

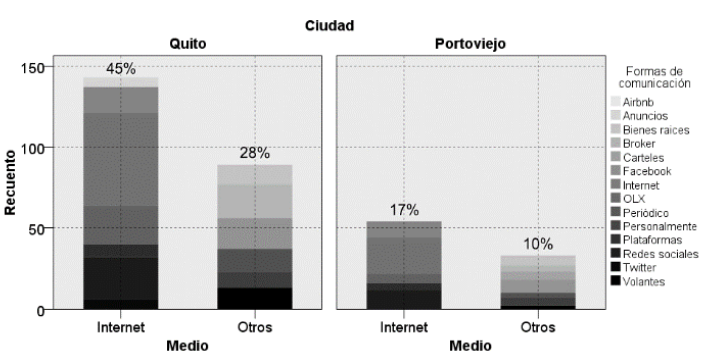

Fig. 6: Internet y Otros - Quito y Portoviejo

Fuente: Elaboración propia

El gráfico 6 establece la participación mayoritaria del internet entre ambas ciudades por encima del $64 \%$, frente a un menor y no ausente uso de los medios tradicionales para la oferta de vivienda en el Ecuador. Consecuentemente, se evidencia que el uso del internet para ofertar vivienda por parte de propietarios de la misma es el medio más usado.

\section{CONCLUSIÓN}

En base al análisis de resultados, se evidencia que el genero masculino tuvo mayor participación en esta investigación dirigida a propietarios de vivienda en venta o alquiler. Así también, el rango de edad más representativo de los participantes en la encuesta fue de 25 a 40 años; demostrando que las nuevas generaciones son las que en su mayoría utilizan la tecnología.

Por su parte, los ciudadanos de ambas ciudades y de edades comprendidas entre 50 y 60, reportan el uso habitual de medios tradicionales, sin embargo, usan la internet en menor proporción, sin descartar su potencial alcance a niveles de uso identificados en rangos de menor edad. La conectividad y uso de TICs, en este rango de edad, es referencia para nuevas investigaciones, centradas en el análisis de los factores que inciden en el uso diferenciado de la tecnología y su promoción como elementos que apuntan al conectivismo.

Se puede inferir que la tendencia por parte de personas que no usan hacen uso de las tecnologías de comunicación mediante redes interconectadas y plataformas web, podría 
ir en aumento, por ende, a su participación en el conectivismo.

Se ha demostrado que el uso de medios tecnológicos como el Internet, Redes sociales como facebook, ; y plataformas como olx contribuyen predominantemente para ofertar vivienda en las ciudades de Quito y Portoviejo, por parte de propietarios de la misma. El alto uso de tecnologías para ofrecer sus viviendas, demuestra un alto nivel de participación ciudadana en el conectivismo.

\section{REFERENCIAS}

[1] A. M. Townsend, "Smart cities: Big data, civic hackers, and the quest for a new utopia." WW Norton Company, 2013.

[2] OECD, "Digital economy data highlights." Digital Economy Data Highlights, 2016.

[3] OECD, "Stimulating digital innovation for growth and inclusiveness the role of policies for the successful diffusion of ict 2016," in Ministerial meeting on the digital economy background, (Paris), OECD Publishing, 2016.

[4] E. Portmann, M. E. Tabacchi, R. Seising, and A. E. Habenstein, Designing Cognitive Cities. Springer, 2019.

[5] F. Güell and J. M., "Planificación estratégica de las ciudades. nuevos instrumentos y procesos," Investigaciones Regionales, vol. 10, pp. 269-275, 2019.

[6] E. Portmann and F. M, Towards cognitive cities: Advances in cognitive computing and its applications to the governance of large urban systems. Berlin: Springer, 2016.
[7] A. Abdelnaiem, A. Mohamed, and B. National, International Alumni Seminar on Smart City Towards Human Smart Cities. 2018.

[8] H. Kumar, M. K. Singh, M. P. Gupta, and J. Madaan, Moving towards smart cities: Solutions that lead to the Smart City Transformation Framework. Technological Forecasting and Social Change. 2018.

[9] V. Beinrott, Bürgerorientierte Smart City: Potentiale und Herausforderungen; Monographie am The Open Government Institutel TOGI der ZeppelinUniversit. ${ }^{a}$. Epubli, 2015.

[10] G. Siemens, "Connectivism: A learning theory for the digital age. elearnspace." 2006.

[11] A. Abdul, J. Vermeulen, D. Wang, B. Y. Lim, and M. Kankanhalli, Trends and Trajectories for Explainable, Accountable and Intelligible Systems: An HCI Research. 2018.

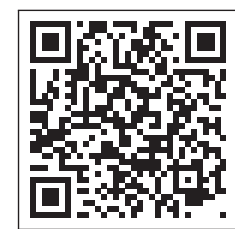


